\title{
Synthesis and Antimicrobial Evaluation of Some Novel 2-(4-Chlorophenylimino) thiazolidin-4-one Derivatives
}

\author{
H. B'Bhatt and S. Sharma* \\ Department of Chemistry, Hemchandracharya North Gujarat University, Patan-384 265, Gujarat, India \\ ${ }^{*}$ E-mail: sangitamem2000@gmail.com
}

(Received February 6, 2012; Accepted May 7, 2012)

\begin{abstract}
A series of 2-(4-chlorophenylimino)-5-((3-( $p$-substituted phenyl)-1-phenyl-1H-pyrazol-4-yl) methylene) thiazolidin-4-one (3a-h) compounds were prepared from the 2-(4-chlorophenylimino) thiazolidin-4-one (1) and 1-phenyl-3-( $p$ substituted phenyl)-1H-pyrazole-4-carbaldehyde (2a-h). All compounds were characterized by elemental (C, H, N) analysis and spectral (FT-IR, ${ }^{1} \mathrm{H}$ NMR and GC-MS) analysis. These newly synthesized compounds were screened for their antibacterial and antifungal activities. Antimicrobial activity was observed and evaluated against the bacterial strains like Eschericha coli (MTCC 443), Pseudomonas aeruginosa (MTCC 1688), Staphylococcus aureus (MTCC 96), Streptococcus pyogenes (MTCC 442) and against the fungal strains like Candida albicans (MTCC 227), Aspergillus niger (MTCC 282) and Aspergillus clavatus (MTCC 1323). All the synthesized compounds were found to possess moderate to excellent antimicrobial activity against above selected strains.
\end{abstract}

Key words: 2-(4-Chlorophenylimino) thiazolidin-4-one, Antibacterial activity, Anti fungal activity, Vilsmeier-Haack reaction, Knoevenagel condensation reaction

\section{INTRODUCTION}

For more than a century, heterocyclic compounds are known to have significant and important place in organic chemistry. These compounds are the part and parcel of many important biochemical processes and are the constituents of main compounds like DNA, RNA, amino acids, purines, pyrimidine bases and vitamins in live cells. It has been established that more than half of the therapeutic agents available till date consist of sulphur and/ or nitrogen containing heterocyclic moieties and these have dragged the attention of chemists due to their broad spectrum biological activities and usage in various fields of pharmacy.

The biological and therapeutic properties of thiazolidinone have immensely initiated the synthesis of many derivatives of this moiety. 4-thiazolidinone derivatives are well known for their variety of pharmacological properties such as antibacterial, ${ }^{1}$ anticonvulsant, ${ }^{2}$ sedative, ${ }^{3}$ antimycobacterial, ${ }^{4,5}$ anti-tuberculosis, ${ }^{6,7}$ anti-inflammatory, ${ }^{8-10}$ anti-HIV, ${ }^{11-13}$ anti tumor, ${ }^{14}$ anti diabetic activity. ${ }^{15,16}$

It is always interesting for medicinal chemist to synthesize more potent compounds. Various derivatives of pyrazoles are already synthesized and screened for various pharmacological activities like antimicrobial, ${ }^{17-21}$ anticonvulsant, ${ }^{22}$ anti inflammatory, ${ }^{23-25}$ antimalerial $^{26}$ and antiviral activity. $^{27}$

Looking to the importance of above mentioned thiazolidin-4-one derivatives, we intend to synthesize new entities like 2-(4-chlorophenylimino)-5-((3-( $p$-substituted phenyl)-1phenyl-1H-pyrazol-4-yl)methylene)thiazolidin-4-one (3a-h) using 2-(4-chlorophenylimino) thiazolidin-4-one (1) and 1-phenyl-3-( $p$-substituted phenyl)-1H-pyrazole-4-carbaldehyde (2a-h).

\section{RESULTS AND DISCUSSION}

\section{Chemical Characterization}

In the present investigation, we have synthesized, characterized the novel 2-(4-chlorophenylimino)-5-((3-( $p$ substitutedphenyl)-1-phenyl-1H-pyrazol-4-yl)methylene) thiazolidin-4-one (3a-h) derivatives and studied their antimicrobial activity. 4-chloro aniline was reacted with ammonium thiocyanate to produce 4-Chlorophenyl thiourea, which was further reacted with mono chloro acetic acid to produce 2-(4-chlorophenylimino) thiazolidin-4-one (1). 1-phenyl-3-( $p$-substituted phenyl)-1H-pyrazole-4-carbaldehyde (2a-h) compounds were synthesized from $p$ substituted acetophenone, phenyl hydrazine, phosphorous oxychloride and formaldehyde using the Vilsmeier-Haack reaction. Substituted acetophenone was reacted with phenyl hydrazine to produce hydrazones, which was further 


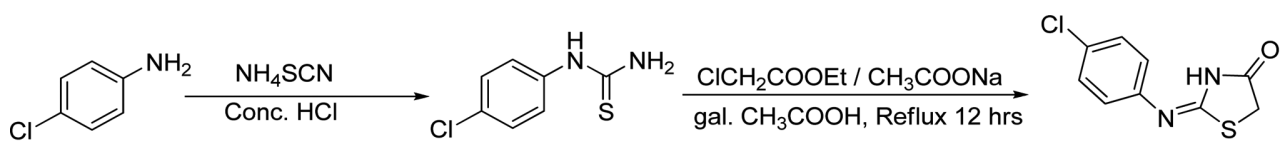

(1)
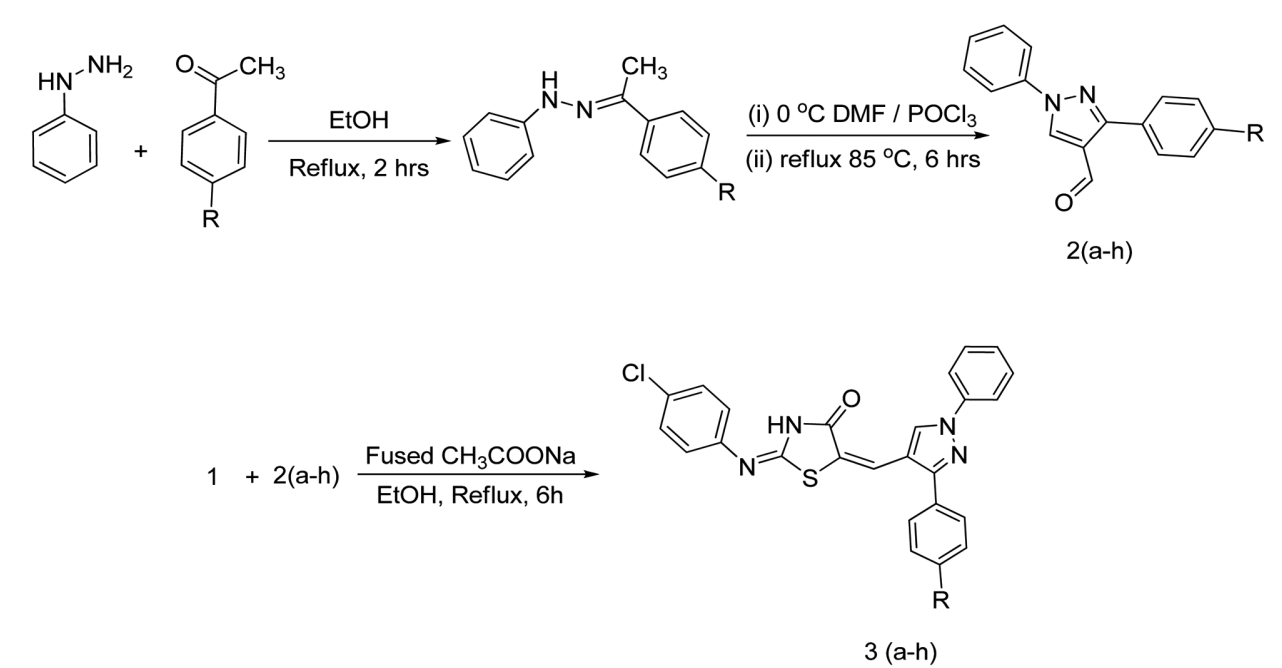

Where $\mathrm{R}=-\mathrm{H}$, or $-\mathrm{CH}_{3}$, or $-\mathrm{OH}$, or $-\mathrm{NO}_{2}$, or $-\mathrm{F}$, or $-\mathrm{Br}$, or $-\mathrm{Cl}$, or $-\mathrm{OCH}_{3}$

Scheme 1. Schematic representation of synthesis of 2-(4-chlorophenylimino)-5-((3-(p-substituted phenyl)-1-phenyl-1H-pyrazol-4-yl) methylene) thiazolidin-4-one (3a-h).

reacted with formylating solution (Dimethyl formamide and phosphorous-oxychloride solution) to produce 1-phenyl-3-( $p$-substituted phenyl)-1 $H$-pyrazole-4-carbaldehyde. 2-(4-chlorophenylimino) thiazolidin-4-one (1). 1-phenyl3-(p-substituted phenyl)-1H-pyrazole-4-carbaldehyde (2a-h) were subjected to Knoevenagel condensation reaction to produce 2-(4-chlorophenylimino)-5-((3-( $p$-substituted phenyl)-1-phenyl-1 $H$-pyrazol-4-yl) methylene) thiazolidin4-one (3a-h) (Scheme 1). All the synthesized compounds were characterized by elemental analysis, FT-IR, NMR and Mass spectroscopy. The IR Spectra of synthesized compounds (3a-h) is interpreted by assigning expected bands to the synthesized compounds. The band observed around $1360-1320 \mathrm{~cm}^{-1}$ is attributed to $\mathrm{C}=\mathrm{S}$ stretching vibration of thiazole ring and bands at $\sim 1600-1510 \mathrm{~cm}^{-1}$ is attributed to $\mathrm{C}=\mathrm{N}$ stretching vibration of pyrazole moiety. Strong band in the region $1725-1670 \mathrm{~cm}^{-1}$ is attributed to $-\mathrm{C}=\mathrm{O}$ stretching of thiazolidinone ring. ${ }^{1} \mathrm{H}-\mathrm{NMR}$ and Mass spectral data of compound (3a-h) are shown in characterization data of Experimental section. Chemical shift $\delta$ at around 8.66-8.60 ppm is due to the presence of $\mathrm{C}=\mathrm{CH}$ group in pyrazole ring. The entire series compounds were quantitatively analyzed for $\mathrm{C}, \mathrm{H}$, and $\mathrm{N}$ and results were found satisfactorily.

\section{Antimicrobial Evaluation}

Antimicrobial drugs known for high potential therapeutic characteristics are always in demand. To establish newly synthesized entities as potential therapeutic candidates appropriate scientific loom, perfect experimental set ups and precisely planned execution of methods is required. Antimicrobial activity of synthesized compounds was performed against four different bacterial strains like, E.coli, P.aeruginosa, S.aureus and S.pyogenes and three different fungi like, C.albicans, A.niger and A.clavatus. Ampicillin and Griseofulvin were used as positive control drugs for selected bacteria and fungi respectively. Solvent control was also studied to know the effect of solvent on activity on selected microorganisms.

\section{Antibacterial Activity}

The results of antibacterial screening of newly synthesized compounds are presented in Table 1. Results reveal that four selected bacterial strains like, E. coli, P. aeruginosa, S. aureus and S. pyogenes has shown different patterns of activities against the control drugs. For, E. coli, compound $3 \mathrm{e}\left(\mathrm{R}^{\prime}=4-\mathrm{F}\right)$ possessed good activity, while compounds $3 \mathrm{c}, 3 \mathrm{~d}, 3 \mathrm{f}$ and $3 \mathrm{~h}\left(\mathrm{R}^{\prime}=4-\mathrm{OH}, 4-\mathrm{NO}_{2}, 4-\mathrm{Br}\right.$ and $4-\mathrm{OCH}_{3}$ respectively) possessed moderate activity when 
Table 1. Antibacterial and Antifungal activity of compounds (3a-h)

\begin{tabular}{|c|c|c|c|c|c|c|c|c|}
\hline & & \multicolumn{4}{|c|}{ Bacterial Strains } & \multicolumn{3}{|c|}{ Fungal Strains } \\
\hline & & $\begin{array}{c}\text { E. coli } \\
\text { (MTCC 443) }\end{array}$ & $\begin{array}{l}\text { P. aeruginosa } \\
\text { (MTCC 1688) }\end{array}$ & $\begin{array}{c}\text { S. aureus } \\
\text { (MTCC 96) }\end{array}$ & $\begin{array}{l}\text { S. pyogenes } \\
\text { (MTCC 442) }\end{array}$ & $\begin{array}{l}\text { C. albicans } \\
\text { (MTCC 227) }\end{array}$ & $\begin{array}{c}\text { A. niger } \\
\text { (MTCC 282) }\end{array}$ & $\begin{array}{c}\text { A. clavatus } \\
\text { (MTCC 1323) }\end{array}$ \\
\hline Comp. Code & $-\mathrm{R}$ & \multicolumn{7}{|c|}{ Minimum Inhibition Concentration (MIC) $\mu \mathrm{g} / \mathrm{ml}$} \\
\hline $3 \mathrm{a}$ & $-\mathrm{H}$ & 500 & 500 & 200 & 200 & 250 & 500 & $>1000$ \\
\hline $3 b$ & $-\mathrm{CH}_{3}$ & 500 & 500 & 250 & 250 & 500 & 500 & 1000 \\
\hline $3 \mathrm{c}$ & $-\mathrm{OH}$ & 250 & 250 & 500 & 500 & 1000 & $>1000$ & $>1000$ \\
\hline $3 d$ & $-\mathrm{NO}_{2}$ & 200 & 250 & 1000 & 1000 & 250 & 500 & 500 \\
\hline $3 e$ & $-F$ & 100 & 100 & 250 & 500 & 500 & 500 & 500 \\
\hline $3 f$ & $-\mathrm{Br}$ & 250 & 250 & 500 & 500 & 500 & $>1000$ & $>1000$ \\
\hline $3 g$ & $-\mathrm{Cl}$ & 500 & 250 & 500 & 500 & 250 & 500 & 1000 \\
\hline $3 \mathrm{~h}$ & $-\mathrm{OCH}_{3}$ & 250 & 200 & 200 & 100 & 100 & 500 & 500 \\
\hline \multicolumn{2}{|c|}{ Ampicillin } & 100 & 100 & 250 & 100 & & & \\
\hline \multicolumn{2}{|c|}{ Griseofulvin } & & & & & 500 & 100 & 100 \\
\hline
\end{tabular}

compared to the standard drug ampicillin. In case of $P$. aeruginosa, compound $3 \mathrm{e}\left(\mathrm{R}^{\prime}=4-\mathrm{F}\right)$ showed good activity while compounds $3 \mathrm{c}, 3 \mathrm{~d}, 3 \mathrm{f}, 3 \mathrm{~g}$ and $3 \mathrm{~h}\left(\mathrm{R}^{\prime}=4-\mathrm{OH}, 4-\right.$ $\mathrm{NO}_{2}, 4-\mathrm{Br}, 4-\mathrm{Cl}$ and4- $\mathrm{OCH}_{3}$ respectively) showed moderate activity when compared to the standard drug ampicillin. Compounds $3 \mathrm{~d}$ possessed excellent activity and compounds $3 \mathrm{a}, 3 \mathrm{~b}$, $3 \mathrm{e}$ and $3 \mathrm{~h}\left(\mathrm{R}^{\prime}=4-\mathrm{H}, 4-\mathrm{CH}_{3}, 4-\mathrm{F}\right.$ and $4-$ $\mathrm{OCH}_{3}$ respectively) possessed good activity when compared to the standard drug ampicillin in case of $S$. aureus. For, $S$. pyogenes, compound $3 \mathrm{~h}\left(\mathrm{R}^{\prime}=4-\mathrm{OCH}_{3}\right)$ possessed good activity while compound $3 \mathrm{a}$ and $3 \mathrm{~b}\left(\mathrm{R}^{\prime}=4-\mathrm{H}\right.$ and 4$\mathrm{CH}_{3}$ ) possessed moderate activity as compared to the standard drug ampicillin. Out of all the synthesized compounds, compound $3 \mathrm{~h}$ showed good activity for all bacterial strains as compared to parent compounds 1 and $2 \mathrm{~g}$ (Fig. 1). However the other synthesized compounds showed specificity towards a particular microbial strain thereby revealing a random inhibition potential pattern

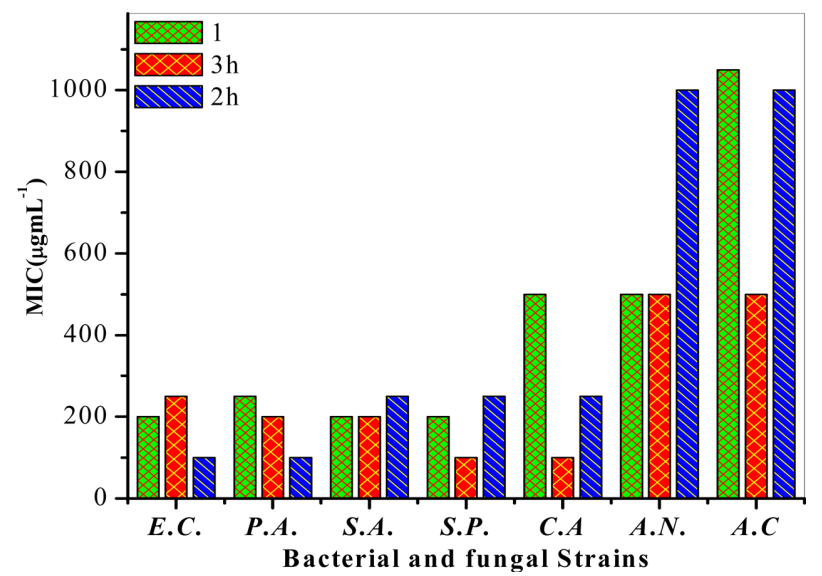

Fig. 1. Comparative Study of Antibacterial activity for compounds $3 \mathrm{~h}, 2 \mathrm{~h}$ and 1. when compared with their parental compounds ( 1 and $2 \mathrm{~g}$ ) (Table 2).

\section{Antifungal Activity}

Antifungal screening of data of newly synthesized compounds are reported in Table 1. To carryout antifungal study, three different fungal strains like C. albicans, A. niger, and A. clavatus were selected. For, C. albicans, compound $3 \mathrm{~h}$ $\left(\mathrm{R}^{\prime}=4-\mathrm{OCH}_{3}\right)$ possessed excellent activity, compounds $3 \mathrm{a}$, $3 \mathrm{~d}$ and $3 \mathrm{~g}\left(\mathrm{R}^{\prime}=4-\mathrm{H}, 4-\mathrm{NO}_{2}\right.$ and $4-\mathrm{Cl}$ respectively) possessed very good activity, compounds $3 \mathrm{~b}, 3 \mathrm{e}$ and $3 \mathrm{f}$ $\left(\mathrm{R}^{\prime}=4-\mathrm{CH}_{3}, 4-\mathrm{F}\right.$ and $4-\mathrm{Br}$ respectively) possessed good activity, while compounds $3 \mathrm{~b}, 3 \mathrm{e}$ and $3 \mathrm{f}\left(\mathrm{R}^{\prime}=4-\mathrm{CH}_{3}, 4-\mathrm{F}\right.$ and $4-\mathrm{Br}$ respectively) possessed moderate activity as compared to the standard drug, Griseofulvin. Amongst all the synthesized compounds, compound $3 \mathrm{~d}\left(\mathrm{R}^{\prime}=4-\mathrm{NO}_{2}\right) 3 \mathrm{~g}$ $\left(\mathrm{R}^{\prime}=4-\mathrm{Cl}\right)$ and $3 \mathrm{~h}\left(\mathrm{R}^{\prime}=4-\mathrm{OCH}_{3}\right)$ showed good to very good activity for all fungal strains compared to parent compounds (1 and 2g) (Fig. 1). However, the other synthesized compounds showed specificity towards particular microbial strain thereby revealed random inhibition potential behavior when compared to their parental compounds (1 and 2g) (Table 2).

\section{CONCLUSION}

Novel series of 2-(4-chlorophenylimino)-5-((3-( $p$-substituted phenyl)-1-phenyl-1 $H$-pyrazol-4-yl)methylene)thiazolidin-4-one (3a-h) compounds were synthesized from 2(4-chlorophenylimino) thiazolidin-4-one (1) and 1-phenyl-3-( $p$-substituted phenyl)-1H-pyrazole-4-carbaldehydes (2a-h). Analytical and spectral data obtained (CHN/S and FT-IR, ${ }^{1} \mathrm{H}-\mathrm{NMR}, \mathrm{GC}-\mathrm{MS}$ ) for all the synthesized com- 
Table 2. Antibacterial and Antifungal activity of the synthesized compounds [(1) and (2a-h)]

\begin{tabular}{|c|c|c|c|c|c|c|c|c|}
\hline & & \multicolumn{4}{|c|}{ Bacterial Strains } & \multicolumn{3}{|c|}{ Fungal Strains } \\
\hline & & $\begin{array}{c}\text { E. coli } \\
\text { (MTCC 443) }\end{array}$ & $\begin{array}{l}\text { P. aeruginosa } \\
\text { (MTCC 1688) }\end{array}$ & $\begin{array}{c}\text { S. aureus } \\
\text { (MTCC 96) }\end{array}$ & $\begin{array}{l}\text { S. pyogenes } \\
\text { (MTCC 442) }\end{array}$ & $\begin{array}{l}\text { C. albicans } \\
\text { (MTCC 227) }\end{array}$ & $\begin{array}{c}\text { A. niger } \\
\text { (MTCC 282) }\end{array}$ & $\begin{array}{c}\text { A. clavatus } \\
\text { (MTCC 1323) }\end{array}$ \\
\hline Compound Code & $-\mathrm{R}$ & \multicolumn{7}{|c|}{ Minimum Inhibition Concentration (MIC) $\mu \mathrm{g} / \mathrm{ml}$} \\
\hline 1 & & 200 & 250 & 200 & 200 & 500 & 500 & $>1000$ \\
\hline $2 \mathrm{a}$ & $-\mathrm{H}$ & 50 & 200 & 200 & 200 & 500 & $>1000$ & $>1000$ \\
\hline $2 \mathrm{~b}$ & $-\mathrm{CH}_{3}$ & 100 & 200 & 200 & 200 & 250 & 500 & 500 \\
\hline $2 \mathrm{c}$ & $-\mathrm{OH}$ & 200 & 200 & 250 & 250 & 500 & 1000 & 1000 \\
\hline $2 d$ & $-\mathrm{NO}_{2}$ & 250 & 250 & 500 & 500 & 500 & $>1000$ & $>1000$ \\
\hline $2 \mathrm{e}$ & $-F$ & 500 & 500 & 250 & 250 & 500 & 1000 & 1000 \\
\hline $2 \mathrm{f}$ & $-\mathrm{Br}$ & 200 & 250 & 100 & 200 & $>1000$ & 500 & 500 \\
\hline $2 \mathrm{~g}$ & $-\mathrm{Cl}$ & 250 & 250 & 250 & 250 & $>1000$ & 200 & 200 \\
\hline $2 \mathrm{~h}$ & $-\mathrm{OCH}_{3}$ & 100 & 100 & 250 & 250 & 250 & 1000 & 1000 \\
\hline \multicolumn{2}{|c|}{ Ampicillin } & 100 & 100 & 250 & 100 & & & \\
\hline \multicolumn{2}{|c|}{ Griseofulvin } & & & & & 500 & 100 & 100 \\
\hline
\end{tabular}

pounds were in full conformity with their proposed structures. Comparison of the antimicrobial results of synthesized compounds (3a-h), it is concluded that the pyrazole derivatives of 2-(4-chlorophenylimino) thiazolidin-4-one (1) has improved their antimicrobial activity when compared with parent compounds. Most of the compounds were found to be active against tested micro-organism. A series of 2-(4chlorophenylimino)-5-((3-( $p$-substituted phenyl)-1-phenyl$1 H$-pyrazol-4-yl) methylene) thiazolidin-4-one compounds (3a-h) exhibited moderate to excellent activity.

\section{EXPERIMENTAL}

Laboratory chemicals used were supplied by Ranbaxy Ltd, India. The melting points of the synthesized products were measured using automated Mettler Toledo FP 62 melting point apparatus (Metter Toledo-Switzerland) in open-glass capillary and were reported uncorrected. Compound purity was checked by thin layer chromatography (TLC). Ethyl acetate: methanol (1:9) solvent system was used as mobile phase in TLC and the spots were monitored under simple UV light chamber. The FT-IR spectra of synthesized compounds were recorded using KBr-pallets technique on a Perkin Elmer - Spectrum GX FT-IR System (PE-USA) in range of 4000-400 $\mathrm{cm}^{-1} .{ }^{1} \mathrm{H}$ spectra were recorded using $200 \mathrm{MHz}$ Bruker Avance DPX NMR system. Chemicals shifts were expressed in $\delta$ (ppm) relative to Tetramethylsilane (TMS) as an internal standard using DMSO- $d_{6}$ as solvent. Elemental analyses of these novel compounds were performed on Perkin Elmer-2400 CHNS/O analyzer (PE-USA). Results of elemental analysis of all compounds were in well agreement with the theoretical values. The mass spectra were scanned with the help of sophisticated Shimadzu QP2010 spectrometer (Shimadzu-Japan), which was equipped with a direct inlet probe and mass detector system which was operated at $70 \mathrm{eV}$.

\section{Biological Assay \\ Antibacterial activity:}

The novel compounds were evaluated with different bacterial and fungal strains. Antibacterial activity was tested against gram positive bacteria Staphylococcus aureus (MTCC-96) and Streptococcus pyogenes (MTCC-442) and gram negative Escherichia coli (MTCC-443) and Pseudomonas aeruginosa (MTCC-1688). Thiazoles are known for inhibiting protein synthesis in bacteria by binding to the complex formed between 23S rRNA and ribosomal protein L11, thereby restricting the action of GTP dependent elongation factors. ${ }^{30}$ Antibacterial activity was carried out by serial broth dilution method. ${ }^{31}$ The standard strains used for the antimicrobial activity were procured from Institute of Microbial Technology, Chandigarh, India. The compounds (3a-h) were screened for their antibacterial activity in triplicate against Escherichia coli, Staphylococcus aureus, Pseudomonas aeruginosa, and Streptococcus pyogenes at different concentrations of 1000 , $500,200,100,50,25,12.5 \mu \mathrm{g} / \mathrm{ml}$ as shown in (Table 1). The novel compounds which were found to be active in primary screening were diluted to obtain $100,50,25,12.5$ $\mu \mathrm{g} / \mathrm{ml}$ concentrations. The growths of bacterial cultures were monitored after 24 and $48 \mathrm{~h}$. The lowest concentration, which showed no growth after spot subculture was considered as MIC for each drug. The highest dilution showing at least $99 \%$ inhibition is taken as MIC. The test 
mixture contained $\sim 10^{8}$ cells $/ \mathrm{ml}$. The standard drug used in this study was 'ampicillin' and it showed MIC at 100, 100,250 , and $100 \mu \mathrm{g} / \mathrm{ml}$ for evaluating antibacterial activity against $E$. coli, $P$. aeruginosa, $S$. aureus and $S$. pyogenes respectively.

\section{Antifungal activity:}

Same compounds were tested for antifungal activity in triplicate against Candida albicans, Aspergillus niger, and Aspergillus clavatus at various concentrations of 1000 , 500,200 , and $100 \mu \mathrm{g} / \mathrm{ml}$ as shown in (Table 1). The results were recorded in the form of primary and secondary screening. The synthesized compounds were diluted to $1000 \mu \mathrm{g} / \mathrm{ml}$ as a stock solution. The synthesized compounds which were found to be active in this primary screening were further tested in a second set of dilution against all microorganisms. The lowest concentration, which showed no growth after spot subculture was considered as MIC for each drug and the highest dilution showing at least $99 \%$ inhibition wass taken as MIC. The test mixture contained $\sim 10^{8}$ spores/ml. "Griseofulvin" was used as a standard drug for antifungal activity and its MIC values are at 500, 100 and $100 \mu \mathrm{g} / \mathrm{ml}$ for Candida albicans, Aspergillus niger, and Aspergillus clavatus, respectively.

\section{General Synthetic Methods}

(i) Synthesis of 2-(4-chlorophenylimino) thiazolidin4-one (1): The titled compound was prepared according to method reported earlier. ${ }^{28}$

(ii) Synthesis of 1-phenyl-3-(p-substituted phenyl)1H-pyrazole-4-carbaldehydes (2a-h): Was carried out according to methods given in literature. ${ }^{29}$

(iii) Synthesis of 2-(4-chlorophenylimino)-5-((3-(psubstituted phenyl)-1-phenyl-1H-pyrazol-4-yl) methylene) thiazolidin-4-one (3a-h): To a solution of 2-(4chlorophenylimino)thiazolidin-4-one (1) $(10 \mathrm{mM})$ in ethanol and anhydrous sodium acetate $(10 \mathrm{mM}), 1$-phenyl-3( $p$ substituted phenyl)-1H-pyrazole-4-carbaldehyde (2a-h) $(10 \mathrm{mM})$ was added. The reaction mixture was heated and refluxed for $6 \mathrm{~h}$. A bright yellow crystalline product was formed and the excess solvent was removed at reduced pressure. Crude product was washed with plenty of water, isolated by filtration and re-crystallized from ethanol to get compounds (3a-h).

\section{Characterization Data}

(i) 2-(4-chlorophenylimino)-5-((1,3-diphenyl-1H-pyrazol-4-yl)methylene)thiazolidin-4-one (3a): Yield 72\%; Yellow crystalline solid; mp 214-216 ${ }^{\circ} \mathrm{C}$; IR $\left(\mathrm{KBr}, \mathrm{cm}^{-1}\right)$ v: 3408 (NH- Stretching), 3120, 2995 (Ar-H stretching, pyrazole $-\mathrm{H}$ stretching), 1707 ( $\mathrm{C}=\mathrm{O}$ stretching), 1536, 1496, $1445(\mathrm{C}=\mathrm{N}, \mathrm{C}=\mathrm{C}$, aromatic ring), $1340(\mathrm{C}=\mathrm{S}$ stretching), 693 (C-S-C linkage); ${ }^{1} \mathrm{H}$ NMR (DMSO) $\delta$ (ppm): 8.65 (s, $1 \mathrm{H},-\mathrm{C}=\mathrm{CH}$ group in pyrazole ring), 8.18-7.06 $(\mathrm{m}, 16 \mathrm{H}$, Ar-H, C-NH, -C=CH ); MS: m/z: $456\left(\mathrm{M}^{+}\right), 305,276,215$, 172; Analytical calculations for $\mathrm{C}_{25} \mathrm{H}_{17} \mathrm{ClN}_{4} \mathrm{OS}$ (456.0) (\%): C, 65.71; H, 3.75; N, 12.26; S, 7.02; Found: C, 65.58; H, 3.67; N, 12.32; S, 7.12.

(ii) 2-(4-chlorophenylimino)-5-((1-phenyl-3-p-tolyl-1Hpyrazol-4-yl)methylene)thiazolidin-4-one (3b): Yield 79\%; Yellow crystalline solid; mp $154-156^{\circ} \mathrm{C}$; IR $\left(\mathrm{KBr}, \mathrm{cm}^{-1}\right)$ v: 3417 (NH- Stretching), 3120, 3060 (Ar-H stretching, pyrazole $-\mathrm{H}$ stretching), $1711(\mathrm{C}=\mathrm{O}$ stretching), 1521, 1491, $1451(\mathrm{C}=\mathrm{N}, \mathrm{C}=\mathrm{C}$, aromatic ring), $1359(\mathrm{C}=\mathrm{S}$ stretching), 685 (C-S-C linkage); ${ }^{1} \mathrm{H}$ NMR (DMSO ) $\delta$ (ppm): $8.61(\mathrm{~s}, 1 \mathrm{H},-\mathrm{C}=\mathrm{CH}$ group in pyrazole ring), 8.08-7.05 (m, $15 \mathrm{H}, \mathrm{Ar}-\mathrm{H}, \mathrm{C}-\mathrm{NH},-\mathrm{C}=\mathrm{CH}), 2.38\left(\mathrm{~s}, 3 \mathrm{H}, \mathrm{Ar}^{-\mathrm{CH}_{3}}\right.$ in pyrazole ring); MS: m/z: $469\left(\mathrm{M}^{+}\right), 438,276,215,122$; Analytical calculations for $\mathrm{C}_{26} \mathrm{H}_{19} \mathrm{ClN}_{4} \mathrm{OS}$ (470.0) (\%): C, 66.30; H, 4.07; N, 11.90; S, 6.81; Found: C, 66.12; H, 4.17 ; N, 11.77; S, 6.96.

(iii) 2-(4-chlorophenylimino)-5-((3-(4-hydroxyphenyl)1-phenyl-1H-pyrazol-4-yl)methylene) thiazolidin-4-one (3c): Yield 82\%; Yellow crystalline solid; $m p 210-212{ }^{\circ} \mathrm{C}$; IR $\left(\mathrm{KBr}, \mathrm{cm}^{-1}\right.$ ) v: 3343 (NH- Stretching), 3110, 3066 (Ar$\mathrm{H}$ stretching, pyrazole $-\mathrm{H}$ stretching $), 1683(\mathrm{C}=\mathrm{O}$ stretching), 1519, 1492, $1447(\mathrm{C}=\mathrm{N}, \mathrm{C}=\mathrm{C}$, aromatic ring), 1358 $\left(\mathrm{C}=\mathrm{S}\right.$ stretching), 688 (C-S-C linkage); ${ }^{1} \mathrm{H}$ NMR (DMSO) $\delta(\mathrm{ppm}): 8.61(\mathrm{~s}, 1 \mathrm{H},-\mathrm{C}=\mathrm{CH}$ group in pyrazole ring), 7.96-7.04 (m, 16H, Ar-OH, C-NH, -C=CH ); MS: m/z: $472\left(\mathrm{M}^{+}\right), 276,215,173,140$; Analytical calculations for $\mathrm{C}_{25} \mathrm{H}_{17} \mathrm{ClN}_{4} \mathrm{O}_{2} \mathrm{~S}$ (472.0) (\%): C, 63.49; H, 3.62; N, 11.85; S, 6.78; Found: C, 63.32; H, 3.48; N, 11.65; S, 6.61.

(iv) 2-(4-chlorophenylimino)-5-((3-(4-nitrophenyl)1-phenyl-1H-pyrazol-4-yl)methylene) thiazolidin-4-one (3d): Yield 68\%; Yellow crystalline solid; $\mathrm{mp} 138-140^{\circ} \mathrm{C}$; IR $\left(\mathrm{KBr}, \mathrm{cm}^{-1}\right)$ v: 3417 (NH- Stretching), 3122, 3075 (Ar$\mathrm{H}$ stretching, pyrazole $-\mathrm{H}$ stretching), $1712(\mathrm{C}=\mathrm{O}$ stretching), 1531, $1494(\mathrm{C}=\mathrm{N}, \mathrm{C}=\mathrm{C}), 1349(\mathrm{C}=\mathrm{S}$ stretching $), 686$ (C-S-C linkage); ${ }^{1} \mathrm{H}$ NMR (DMSO) $\delta$ (ppm): 8.66 (s, $1 \mathrm{H}$, $-\mathrm{C}=\mathrm{CH}$ group in pyrazole ring), 8.40-7.02 (m, 15H, Ar-H, $\mathrm{C}-\mathrm{NH},-\mathrm{C}=\mathrm{CH})$; MS: m/z: $501\left(\mathrm{M}^{+}\right), 290,257,190,121$; Analytical calculations for $\mathrm{C}_{25} \mathrm{H}_{16} \mathrm{ClN}_{5} \mathrm{O}_{3} \mathrm{~S}$ (501.0) (\%): C, 59.82; H, 3.21; N, 13.95; S, 6.39; Found: C, 59.74; H, $3.22 ; \mathrm{N}, 13.72 ; \mathrm{S}, 6.47$.

(v) 2-(4-chlorophenylimino)-5-((3-(4-fluorophenyl)1-phenyl-1H-pyrazol-4-yl)methylene) thiazolidin-4-one (3e): Yield $74 \%$; Yellow crystalline solid; $\mathrm{mp} 283-285^{\circ} \mathrm{C}$; IR $\left(\mathrm{KBr}, \mathrm{cm}^{-1}\right)$ v: 3408 (NH- Stretching), 3123, 3010 (Ar- 
$\mathrm{H}$ stretching, pyrazole $-\mathrm{H}$ stretching $), 1670(\mathrm{C}=\mathrm{O}$ stretching), 1599, 1521, $1450(\mathrm{C}=\mathrm{N}, \mathrm{C}=\mathrm{C}$, aromatic ring $), 1338$ (C=S stretching), 684 (C-S-C linkage), ${ }^{1} \mathrm{H}$ NMR (DMSO) $\delta(\mathrm{ppm}): 8.61(\mathrm{~s}, 1 \mathrm{H},-\mathrm{C}=\mathrm{CH}$ group in pyrazole ring), 7.95-7.05 (m, 15H, Ar-H, C-NH, -C=CH); MS: m/z: 474 $\left(\mathrm{M}^{+}\right)$, 323, 294, 233, 152; Analytical calculations for $\mathrm{C}_{25} \mathrm{H}_{16} \mathrm{ClFN}_{4} \mathrm{OS}$ (474.0) (\%): C, 63.22; H, 3.40; N, 11.80; S, 6.75; Found: C, 63.43; H, 3.25; N, 11.56; S, 6.82.

(vi) 5-((3-(4-chlorophenyl)-1-phenyl-1H-pyrazol-4yl)methylene)-2-(4-chlorophenylimino)thiazolidin-4-one (3f): Yield 79\%; Yellow crystalline solid; mp 134-136 ${ }^{\circ} \mathrm{C}$; IR $\left(\mathrm{KBr}, \mathrm{cm}^{-1}\right)$ v: 3411 (NH- Stretching), 3121, 3025 (Ar$\mathrm{H}$ stretching, pyrazole $-\mathrm{H}$ stretching), $1672(\mathrm{C}=\mathrm{O}$ stretching), 1594, 1521, $1444(\mathrm{C}=\mathrm{N}, \mathrm{C}=\mathrm{C}$, aromatic ring), 1338 (C=S stretching), 684 (C-S-C linkage); ${ }^{1} \mathrm{H}$ NMR (DMSO) $\delta(\mathrm{ppm}): 8.61(\mathrm{~s}, 1 \mathrm{H},-\mathrm{C}=\mathrm{CH}$ group in pyrazole ring), 7.96-7.04 (m, 15H, Ar-H, C-NH, -C=CH ); MS: m/z: 534 $\left(\mathrm{M}^{+}\right)$, 474, 356 , 294, 152; Analytical calculations for $\mathrm{C}_{25} \mathrm{H}_{16} \mathrm{BrClN}_{4} \mathrm{OS}$ (534.0) (\%): C, 56.04; H, 3.01; N, 10.46; S, 5.98;. Found: C, 55.89; H, 3.11; N, 10.54; S, 6.17.

(vii) 2-(4-chlorophenylimino)-5-((3-(4-methoxyphenyl)-1-phenyl-1H-pyrazol-4-yl)methylene)thiazolidin4-one (3g): Yield $87 \%$; Yellow crystalline solid; $\mathrm{mp}>300^{\circ} \mathrm{C}$; IR $\left(\mathrm{KBr}, \mathrm{cm}^{-1}\right)$ v: 3410 (NH- Stretching), 3125, 3000 (Ar$\mathrm{H}$ stretching, pyrazole $-\mathrm{H}$ stretching $), 1700(\mathrm{C}=\mathrm{O}$ stretching), 1596, 1496, $1443(\mathrm{C}=\mathrm{N}, \mathrm{C}=\mathrm{C}$, aromatic ring), 1337 (C=S stretching), 685 (C-S-C linkage); ${ }^{1} \mathrm{H}$ NMR (DMSO) $\delta(\mathrm{ppm}): 8.61$ (s, $1 \mathrm{H},-\mathrm{C}=\mathrm{CH}$ group in pyrazole ring), 7.967.04 (m, 15H, Ar-H, C-NH, -C=CH ); MS: m/z: $490\left(\mathrm{M}^{+}\right)$, 310, 275, 231, 152; Analytical calculations for $\mathrm{C}_{25} \mathrm{H}_{16} \mathrm{Cl}_{2} \mathrm{~N}_{4} \mathrm{OS}$ (490.0) (\%): C, 61.11; H, 3.28; N, 11.40; S, 6.53; Found: C, 61.29; H, 3.34; N, 11.67; S, 6.71.

(viii) 5-((3-(4-chlorophenyl)-1-phenyl-1H-pyrazol-4yl)methylene)-2-(3,4-dichlorophenylimino) thiazolidin4-one (3h): Yield $85 \%$; Yellow crystalline solid; $\mathrm{mp}>300^{\circ} \mathrm{C}$; IR $\left(\mathrm{KBr}, \mathrm{cm}^{-1}\right)$ v: 3401 (NH- Stretching), 3117, 3038 (Ar$\mathrm{H}$ stretching, pyrazole $-\mathrm{H}$ stretching), 1723 ( $\mathrm{C}=\mathrm{O}$ stretching), 1585, 1524, $1438(\mathrm{C}=\mathrm{N}, \mathrm{C}=\mathrm{C}$, aromatic ring), 1331 (C=S stretching), 686 (C-S-C linkage), ${ }^{1} \mathrm{H}$ NMR (DMSO) $\delta(\mathrm{ppm}): 8.61(\mathrm{~s}, 1 \mathrm{H},-\mathrm{C}=\mathrm{CH}$ group in pyrazole ring), 7.96-7.04 (m, 15H, Ar-H, C-NH, $-\mathrm{C}=\mathrm{CH}), 3.84(\mathrm{~s}, 3 \mathrm{H}$, Ar- $-\mathrm{OCH}_{3}$ in pyrazole ring); MS: m/z: $486\left(\mathrm{M}^{+}\right), 335,306$, 263, 152; Analytical calculations for $\mathrm{C}_{26} \mathrm{H}_{19} \mathrm{ClN}_{4} \mathrm{O}_{2} \mathrm{~S}$ (486.0) (\%): C, 64.13; H, 3.93; N, 11.51; S, 6.58; Found: C, 64.29; $\mathrm{H}, 3.82 ; \mathrm{N}, 11.44 ; \mathrm{S}, 6.34$.

Acknowledgements. Authors are thankful to Micro Care laboratory and TRC, Surat, India for carrying out microbial studies.

\section{REFERENCES}

1. Mistry, K. M.; Desai, K. R. E-J. Chem. 2004, 1, 189.

2. Gursoy, A.; Terzioglu, N. Turk. J. Chem. 2005, 29, 247.

3. Knusli, G. E. Chim. Ital. 1949, 79, 621.

4. Kucukguzel, S. G.; Oruc, E. E.; Rollas, S.; Sahin, F.; Ozbek, A. Eur. J. Med. Chem. 2002, 37, 197.

5. Kucukguzel, G.; Kocatepe, A.; Clercq, E. D.; Sahin, F.; Gulluce, M. Eur. J. Med. Chem. 2006, 41, 353.

6. Bukowski, L.; Janowiec, M.; Zwolska-Kwiek, Z.; Andrzejczyk, Z. Pharmaz. 1999, 54, 651.

7. Babaoglu, K.; Page, M. A.; Jones, V. C.; McNeil, M. R.; Dong, C.; Naismith, J. H.; Lee, R. E. Bioorg. Med. Chem. Lett. 2003, 13, 3227.

8. Goel, T.; Ram, R.; Tyagi, E.; Bansal, A.; Kumar, D.; Mukherjee, Sinha, J. N. Eur. J. Med. Chem. 1999, 34, 265.

9. Vigorita, M. G.; Ottana, R.; Monforte, F.; Maccari, R.; Trovato, A.; Monforte, M.; Taviano, M.F. Bioorg. Med. Chem. Lett. 2001, 11, 2791.

10. Bhati, S. K.; Kumar, A. Eur. J. Med. Chem. 2008, 43, 2323.

11. Barreca, M. L.; Chimirri, A.; De Luca, L.; Monforte, A. M.; Monforte, P.; Rao, A.; Zappala, M.; Balzarini, J.; De Clercq, E. Bioorg. Med. Chem. Lett. 2001, 11, 1793.

12. Murugesan, V.; Prabhakar, Y. S.; Katti, S. B. J. Mol. Graph. Model. 2009, 27, 735.

13. Balzarini, J.; Orzeszko-Krzesinska, B.; Maurin, J. K.; Orzesko, A. Eur. J. Med. Chem. 2009, 44, 303.

14. Mosula, L.; Zimenkovsky, B.; Havrylyuk, D.; Missir, A. V.; Chirita, I. C.; Lesyk, R. Farmac. 2009, 57, 321.

15. Jeo, R.; Park, S. Arch. Pharm. Res. 2004, 27, 1099.

16. Lesyk, R.; Vladzimirska, O.; Zimenkovsky, B. Boll. Chim. Farmace. 1998, 137, 210.

17. Amir, M.; Khan, S.; Khan, M. Ind. J. Heterocycl. Chem. 2001, 11, 55.

18. Jain, R.; Pandya, P.; Bhadauria, J.; Tomar, S. J. Indian. Chem. Soc. 2000, 77, 42.

19. Gupta, D. P.; Bhadauria, R. S.; Soan, V. Int. J. Pharma. Appl. Sci. 2010, 1, 97.

20. Sarma, K. N.; Subha, M. C. S.; Rao, K. C. E-J. Chem. 2010, 7, 745 .

21. Solanki, P. R.; Wadodarkar, K. N. Ind. J. Heterocycl. Chem. 2003, 13, 135.

22. Jr. Owen, J. E.; Swanson, E. E.; Meyers, D. B. J. Am. Pharm. Assoc. 2006, 47, 70.

23. Badawey, E.; El-Ashmawey, I. M. Eur. J. Med. Chem. 1998, 33, 349.

24. Makhsumov, A. G.; Dzhuraev, A. D.; Kilichov, G.; Nikbaev A.T. Khimiko-farmatsevticheskii Zhurnal. 1986, 20, 289.

25. Bekhit, A. A.; Ashour, H. M.; Guemei, A. A. Arch. Pharm. 2005, 338, 167.

26. Dominguez, J. N.; Charris, J. E.; Caparelli, M.; Riggione, F. Arzneimittelforschung 2002, 52, 482.

27. Ouyang, G.; Chen, Z.; Cai, X.; Song, B.; Bhadury, P. S.; 
Yang, S.; Jin, L.; Xue, W.; Hu, D.; Zeng, S. Bioorg. Med. Chem. 2008, 16, 9699.

28. Zhou, H.; Wu, S.; Zhai, S.; Liu, A.; Sun, Y.; Li, R.; Zhang, Y.; Ekins, S.; Swaan, P. W.; Fang, B.; Zhang, B.; Yan, B. J. Med. Chem. 2008, 51, 1242.

29. Pier, G. B.; Barbara, C.; Giampiero, S.; Romeo, R.; Gio- vanni, B.; Abdel, N. Z.; Maria, J.; de las, I. Synthesis 1997, 10, 1140.

30. Porse, B. T.; Leviev, L.; Mankin, A. S.; Garret, R. A. J. Mol. Biol. 1998, 276, 391.

31. Ghalem, B. R.; Mohamed, B. Afr. J. Pharm. Pharmacol. 2009, 3, 92 . 